contrast and consistent with the findings reported by Patrick et al., application of short 8-mer oligonucleotides against miR21 did not affect pressure overload-induced cardiac hypertrophy, fibrosis, and cardiac dysfunction. Currently, we do not know why the reported phenotype of the miR-21-deficient mice differs from that of mice that received treatment with long miR-21 inhibitors. Possible reasons are various means of genetic compensation upon constitutive deletion of the Mir21 gene as well as potential off-target effects of anti-miRs that evade current analysis.

Taken together, we confirmed that the 8-mer anti-miR-21 is ineffective in preventing cardiac disease in a mouse model of left ventricular pressure overload, a finding which is likely due to the modest and transient nature of miR-21 suppression by 8 -mers. For long-term inhibition of miR21 function in vivo, interventions based on longer anti-miRs are likely to prove superior, due to their high potency and treatment duration. In contrast, short 8-mer LNAmodified oligonucleotides against miR-21 are of less potency and without therapeutic effects in vivo.

\section{Acknowledgments}

We kindly acknowledge the support of the Deutsche Forschungsgemeinschaft (DFG TH903/10-1), the BMBF (01EO0802 and 01KU0902A), and the Fondation Leducq.

\section{Thomas Thum, ${ }^{1,2}$ Nelson Chau, ${ }^{3}$ Balkrishen Bhat, ${ }^{3}$ Shashi Kumar Gupta, ${ }^{1}$ Peter S. Linsley, ${ }^{3}$ Johann Bauersachs, ${ }^{2}$ and Stefan Engelhardt ${ }^{4}$}

${ }^{1}$ Institute for Molecular and Translational Therapeutic Strategies, IFB-Tx, and ${ }^{2}$ Department of Cardiology and Angiology, Hannover Medical School, Hannover, Germany. ${ }^{3}$ Regulus Therapeutics, San Diego, California, USA. ${ }^{4}$ Institute of Pharmacology and Toxicology, Technische Universitaet Muenchen, Munich, Germany.

Conflict of interest: Thomas Thum, Johann Bauersachs, and Stefan Engelhardt have filed a patent application for the use of miR21 and have received royalty fees through the University of Wuerzburg. Nelson Chau, Balkrishen Bhat, and Peter S. Linsley are employees of Regulus.
Address correspondence to: Thomas Thum, Hannover Medical School, Institute for Molecular and Translational Therapeutic Strategies, Carl-Neuberg-Str. 1, 30625 Hannover, Germany. Phone: 49.511.532.5272; Fax: 49.511.532.5274; E-mail: Thum. Thomas@mh-hannover.de. Or to: Stefan Engelhardt, Institute of Pharmacology and Toxicology, Technische Universitaet Muenchen, Munich, Germany. Phone: 49.89.4140.3260; Fax: 49.89.4140.3261; E-mail: stefan.engelhardt@tum.de.

J Clin Invest. 2011;121(2):461-462. doi:10.1172/JCI45938.

1. Patrick DM, et al. Stress-dependent cardiac remodeling occurs in the absence of microRNA-21 in mice. J Clin Invest. 2010;120(11):3912-3916.

2. Thum T, et al. MicroRNA-21 contributes to myocardial disease by stimulating MAP kinase signalling in fibroblasts. Nature. 2008; 456(7224):980-984.

3. Liu G, et al. miR-21 mediates fibrogenic activation of pulmonary fibroblasts and lung fibrosis. J Exp Med. 2010;207(8):1589-1597.

4. Levin AA, et al. Basic principles of the pharmacokinetics of antisense oligonucleotide drugs. In: Crooke ST, ed. Antisense Drug Technology: Principles, Strategies, and Applications. 2nd ed. Boca Raton, Florida, USA: CRC Press; 2007:183-215.

\title{
Response to Thum et al.
}

$T$ hum et al. conclude that microRNA-21 (miR-21) is essential for cardiac hypertrophy and fibrosis in response to pressure overload (1). They also claim that our failure to observe a blockade to these processes in mice treated with an 8 -mer locked nucleic acid-modified oligonucleotide against miR-21 (called Anti-21) (2) is due to the ineffectiveness of such inhibitors. We wish to point out several caveats to their study regarding the role of miR-21 in cardiac hypertrophy and their conclusions regarding the efficacy of the Anti-21 oligonucleotide.

First, we find that Anti-21 inhibits miR21 with a half-maximal inhibitory concentration of $0.9 \mathrm{nM}$, indicating the efficacy of Anti-21. Second, Thum et al. do not state the method they used to measure miR21 inhibition, though we assume it to be quantitative PCR (qPCR). In our hands, qPCR alone is unreliable for measuring miRNA inhibition, especially for 8-mer inhibitors, since they may be displaced during qPCR and thereby give an underrepresentation of miRNA inhibition. To demon- strate functional inhibition of a miRNA, it is important to show data from multiple assays, such as small RNA Northern blots, luciferase reporter assays, and target derepression, as shown in our study (2). Such data are lacking in the Thum et al. rebuttal, which makes comparison of the different chemistries impossible.

Thum et al. also state that we measured miR-21 inhibition on day 2 after dosing with Anti-21, when in fact we measured inhibition 3 weeks after dosing. At this time point, we observed inhibition of miR-21 in pressure-overloaded hearts at a level significantly below that of control mice. Thus, their approach for inhibition and/or measurement of miR-21 by their 8-mer inhibitors differs markedly from ours, since we observed robust miR-21 inhibition 3 weeks after injection, as demonstrated by multiple readouts (2). Using mismatched oligonucleotide controls is also important for interpreting miRNA inhibition studies in vivo, as described in our paper, rather than using PBS as a control, as reported by Thum et al. (1).
Finally, Thum et al. postulate that constitutive genetic deletion of miR-21 in mice may not reveal the functions of miR-21 in cardiac disease because of compensatory events that mask such functions. If such compensation occurs, it must be specific for the cardiac functions of miR-21, since miR-21 null mice are resistant to lung tumorigenesis (3), consistent with the documented pro-oncogenic functions of miR-21. To further address the possibility of genetic compensation, we have deleted a floxed miR-21 allele immediately prior to thoracic aortic constriction in mice using a ubiquitously expressed tamoxifen-regulated Cre transgene. These animals show cardiac hypertrophy and fibrosis comparable to that of their Cre-negative littermates. Genetic compensation therefore cannot account for the normal pathological cardiac remodeling response in miR-21 null mice. Moreover, functions of other miRNAs in heart disease can be revealed by genetic deletion in mice, as shown for miR-208 (4). Thus, while 22-mer oligonucleotide inhibitors against miR-21 are efficacious in inhib- 
iting cardiac hypertrophy (1), other loss-offunction approaches appear ineffective. We remain enthusiastic about miRNAs as therapeutic targets and welcome such dialog.

\section{David M. Patrick, ${ }^{1}$ Sakari Kauppinen, ${ }^{2}$ Eva van Rooij, ${ }^{3}$ and Eric N. Olson'1}

${ }^{1}$ Department of Molecular Biology, University of Texas Southwestern Medical Center, Dallas, Texas, USA. ${ }^{2}$ Santaris Pharma, Hørsholm, Denmark. ${ }^{3}$ miRagen Therapeutics,
Boulder, Colorado, USA.

Conflict of interest: Eric N. Olson and Eva van Rooij are co-founders of miRagen Therapeutics. Sakari Kauppinen is employed at Santaris Pharma.

Address correspondence to: Eric Olson, University of Texas Southwestern Medical Center, 6000 Harry Hines Blvd., Dallas, Texas 75390-9148, USA. Phone: 214.648.1187; Fax: 214.648.1196; E-mail: Eric.Olson@ utsouthwestern.edu.
J Clin Invest. 2011;121(2):462-463. doi:10.1172/JCI46108.

1. Thum T, et al. MicroRNA-21 contributes to myocardial disease by stimulating MAP kinase signaling in fibroblasts. Nature. 2008;456(7224):980-984.

2. Patrick DM, et al. Stress-dependent cardiac remodeling occurs in the absence of miR-21 in mice. JClin Invest. 2010;120(11):3912-3916.

3. Hatley ME, et al. Modulation of K-Ras-dependent lung tumorigenesis by microRNA-21. Cancer Cell. 2010;18(3):282-293.

4. van Rooij E, Sutherland LB, Qi X, Richardson JA, Hill J, Olson EN. Control of stress-dependent cardiac growth and gene expression by a microRNA. Science. 2007;316(5824):575-579.

\section{Expression of TNFRSF25 on conventional T cells and Tregs}

mmunological tolerance is achieved through recessive and dominant mechanisms. In recessive tolerance the fate of selfreactive $T$ cells is controlled in a cell intrinsic manner such that they undergo cell death or become anergic after exposure to selfantigen. In contrast, dominant tolerance is cell extrinsic and is mediated by $\mathrm{CD}^{+}$ Tregs that express the transcription factor Forkhead P3 (Foxp3) and show increased self-reactivity when compared with conventional T cells (1). Peripheral homeostasis of Tregs is maintained through $\mathrm{T}$ cell receptor stimulation and signaling by IL- 2 and costimulatory receptors such as CD28 $(2,3)$. Identifying the full spectrum of signals controlling Treg homeostasis in vivo may lend itself to therapeutic strategies that can be used to manipulate their number or function in order to ameliorate inflammatory diseases or augment an antitumor immune response. In this regard, the recent study by Schreiber and colleagues in the JCI (4) is particularly interesting, since the authors demonstrate that injection of mice with a mAb that binds to TNF receptor superfamily member 25 (TNFRSF25) selectively expands Tregs. The authors also show that administration of the anti-TNFRSF $25 \mathrm{mAb}$ inhibits a Th2 inflammatory response in the lungs of antigen-sensitized mice. Another interesting observation reported in that study was the preferential binding of the anti-TNFRSF25 mAb to Tregs (4). Based on this, the authors concluded that the level of TNFRSF 25 on Tregs is considerably higher than that found on conventional $\mathrm{CD}^{+}$ $\mathrm{T}$ cells. We have reexamined the expression of TNFRSF 25 on conventional CD $4^{+} \mathrm{T}$ cells and Tregs, and our findings are remarkably different to those reported by Schreiber et al. We used two approaches to investigate expression of TNFRSF25 on T cell subsets (Figure 1). First, we showed that a different anti-TNFRSF25 antibody stains conventional $\mathrm{T}$ cells and Tregs obtained from Foxp3GFP knockin mice (5) with similar intensity (Figure 1, A-C). Second, we demonstrated that soluble recombinant TL1A (sTL1A), the ligand for TNFRSF25, binds equally well to conventional $\mathrm{CD} 4^{+} \mathrm{T}$ cells and Tregs (Figure 1D). We therefore conclude that Tregs and conventional $\mathrm{CD}^{+} \mathrm{T}$ cells express similar levels of functional TNFRSF25.

We agree with the findings of Schreiber et al. that TNFRSF25 triggering can expand Tregs. In fact, we recently showed that transgenic mice that constitutively express TL1A have increased numbers of Tregs (6). However, we also observed increased activation of conventional $\mathrm{CD}^{+} \mathrm{T}$ cells, elevated levels of IL-13 and IL-17, and small intestinal immune pathology that manifests as goblet cell and paneth cell hyperplasia (6). A similar phenotype was reported by Siegel and colleagues using mice that express higher levels of a TL1A transgene (7). Interestingly, we found that stimulation of $\mathrm{T}$ cells with recombinant TL1A attenuates Treg-mediated suppression in vitro and this effect required TNFRSF25 signaling in either conventional CD4 ${ }^{+} \mathrm{T}$ cells or Tregs (6). Thus, TNFRSF25 exerts costimulatory effects on conventional CD $4^{+} \mathrm{T}$ cells as well as on Tregs. Furthermore, its effects on promoting Treg turnover are counterbalanced by its ability to attenuate immune suppression and stimulate effector $\mathrm{T}$ cells. This conclusion is in line with findings obtained using mice that were rendered deficient in either TNFRSF25 or TL1A. In these mice, the net effect of defective TNFRSF25 signaling is a reduction in the severity of T cell-mediated inflammation $(8,9)$.

How then can these findings been reconciled? We suspect that the anti-TNFRSF25 $\mathrm{mAb}$ used by Schreiber et al. may bind to an epitope that is preferentially expressed on Tregs, since multiple mRNA isoforms of TNFRSF25 have been described (10), and this may have lead to preferential expansion of Tregs in their experiments. Indeed, this notion is supported by a previous study showing differences in TNFRSF25 mRNA isoform expression between Tregs and Th17 cells (8). Thus, when compared with conventional T cells, Tregs expressed more TNFRSF25 mRNA transcripts that encode the shorter variant of TNFRSF25, which lacks the fourth extracellular cysteine-rich repeat. It is noteworthy that the antibody used in our study bound to both full-length TNFRSF25 as well as the short variant lacking the fourth cysteine-rich repeat (Figure 1E). We believe that caution should be applied in interpreting the findings obtained with anti-TNFRSF25 antibodies, as these may differ in their capacity to stimulate Tregs and effector $T$ cells.

\section{Acknowledgments}

This work is funded by Leukaemia \& Lymphoma Research.

\section{Vadim Y. Taraban, John R. Ferdinand, and Aymen Al-Shamkhani}

